\title{
Why Do People Buy Virtual Goods: A Meta-Analysis
}

\author{
Authors: Hamari Juho \& Keronen Lauri
}

\begin{abstract}
During the last decade, virtual goods have become an important target of consumption online (especially in games, virtual worlds and social networking services) amongst physical and digital goods. In this study we investigate the question of why do people purchase virtual goods by conducting a metaanalysis of the existing quantitative body of literature (24 studies) on the topic. The meta-analysis revealed an important aspect of value of virtual goods: contrary to traditional goods, the reasons why people purchase virtual goods are tightly connected to the platform where they are sold in. These findings underline the significance of service design and its relationship to the formation of value of virtual goods: the value of virtual goods is context-bound, and therefore, bound to the environment where they are usable in. Most factors that were found to be significant predictors of purchase behavior (such as network effects, selfpresentation, enjoyment, ease of use, flow and use of the platform) are directly related to the aspects and design of the platform beyond the general attitudes towards virtual goods themselves. Moreover, we found that enjoyment and prolonged use of the platform were more important predictors for purchases in virtual worlds than in games.
\end{abstract}

Keywords: Virtual goods, Virtual worlds, Online games, Social networking services, Freemium, Free-toplay 


\section{INTRODUCTION}

Virtual goods have become one of the major categories of consumption in online environments among the purchases of normal physical goods (sold on e.g. Amazon) and digital goods such as music (e.g. iTunes). Virtual goods refer to virtual objects such as items, avatar clothing, weapons, virtual furniture, currencies, characters and tokens that commonly exist solely within variety of virtual environments (Fairfield 2005; Lehdonvirta 2009; Hamari \& Lehdonvirta 2010) where they are usable in. Whereas digital goods such as music and photos can be duplicated, virtual goods are rivalrous implying that they can't be copied but are rather regulated by the rules of the given virtual economy. (Fairfield 2005; Lehdonvirta 2009; Hamari \& Lehdonvirta 2010; Harviainen \& Hamari 2015). Virtual goods are often categorized into appearance, social and functional based goods (e.g. Lehdonvirta 2009). For example, appearance based goods only affect the look of virtual character or avatar whereas functional goods can be service feature unlocks or game items such as more powerful weapons, armor or other boosts that increase various character attributes. Virtual goods are bound by the rules of the environment where they are used. Virtual goods exist within virtual economies, such as in games and virtual worlds (Lehdonvirta \& Castronova 2014). The global virtual goods market value was \$ 14.8 billion in 2012 and was forecasted to continue ascending in near future (TechNavio 2013).

Selling virtual goods has recently become de facto business model for games publishers and many social networking services. Many online games and virtual worlds allow free access to main features and instead rely on generating revenues by selling virtual goods on top of the core service. The topic started to attract academic attention circa 2005 and the first quantitative studies on the motivations to purchase appeared circa 2008. Thus far, studies on topic have been interested in predicting purchase behavior with, for example, experiences related to use of the core service (Animesh et al. 2011; Mäntymäki \& Salo 2013), attractiveness of virtual goods themselves (Kim 
2012; Kim et al. 2011; Wang \& Chang 2014), and desire for self-representation in virtual world (Kim et al. 2011; Kim et al. 2012). Moreover, the topic has been examined from viewpoints of several theoretical perspectives such as technology acceptance (Cha 2011; Domina et al. 2012; Hamari \& Keronen 2016), theories of planned behavior and reasoned action (Gao 2014; Kaburuan et al. 2009), expectancy-disconfirmation model (Wang \& Chang 2013; 2014), as well as transaction cost theory (Guo \& Barnes 2011; 2012). Although this body of literature covers a variety of factors affecting purchase behavior and intention, individual studies are often naturally limited to a singular service, virtual world or a game which restricts generalization of their findings on top of limited sample sizes and inevitable measurement errors. Therefore, the understanding is in need for a comprehensive meta-analysis and synthesis of previous research findings.

In practical and managerial terms, selling virtual goods has become a prominent business model for otherwise free online games (Alha et al. 2014; Hamari 2011; Hamari \& Järvinen 2011; Hamari \& Lehdonvirta 2010; Lehdonvirta 2009; Lehdonvirta \& Castronova 2014; Nieborg 2015) and virtual worlds. Thus, understating why people purchase virtual goods is also a pertinent practical issue for the service operators. Since most of these services have no entry pricing and virtual good purchases are voluntary, it is essential to understand which factors can increase virtual good purchases. In free-to-play games, only a minute percentage of registered users purchase virtual goods, (2\% according to Forbes 2013).

Although selling virtual goods has become powerful business strategy in virtual world and online games, this commodification of games has certainly faced heavy resistance from the users and developers (Alha et al. 2014; Hamari 2015; Kimppa et al. 2016; Lin \& Sun 2011). Many free-toplay game publishers encourage users to purchase functional goods for faster progression and competitive advantage in the game. However, paying for competitive advantage has been regarded as highly incompatible with the nature of games and many players perceive purchasing advantageous goods as cheating. In fact, previous studies have discussed how such purchases can 
decrease engagement, immersion and flow in gameplay experience (Alha et al. 2014; Bartle 2004; Hamari \& Lehdonvirta 2010; Lin \& Sun 2011; Paavilainen et al. 2013). In case competitive balance within a game is desired, understanding why people buy goods for other reasons is essentially important.

This study synthesizes previous research literature meta-analytically and provides estimates for most studied direct correlations with purchasing intention of virtual goods in games and virtual worlds. Since this study aims for as comprehensive as possible literature review of quantitative research and meta-analysis, we are not restricted by theoretical assumptions stemming from any particular theoretical frameworks. We examine the correlations between variables regardless of whether the analyzed studies had modelled a relationship between them in their structural models. Therefore, our study not only presents reliable results on the topic why people purchase virtual goods but is also able to take into account relationships between variables not disclosed as results in prior literature. As the primary objective of this study is to rigorously synthesize and therefore validate and repeat the studies done on the question of why people wish to purchase virtual goods in differing environments, the emphasis of study is to increase the validity and reliability of the empirical results on this area. Therefore, by collating quantitative studies and laying down a reliable foundation for the area of virtual goods consumption motivations, this study act as a reference point for further studies that attempt to take this vein of research further.

\section{PROCEDURE \& METHODS}

This section reports the individual phases in the analysis (see Figure 1). We began the analysis by conducting literature searches, first for defining comprehensive but accurate set of keywords with exploratory searches and then performing formal search of studies. We were required to form criteria for mechanical and unambiguous rejection of unsuitable search hits for analysis. Next, we 
coded the relevant statistics, findings and measures from the remaining set of studies. Then, we conducted descriptive analysis by inspecting sample sizes, virtual environments and theoretical foundations in the included studies. Finally, we validated our approach for random effects model in meta-analysis by testing heterogeneity in the studies and eventually conducted actual meta-analysis.

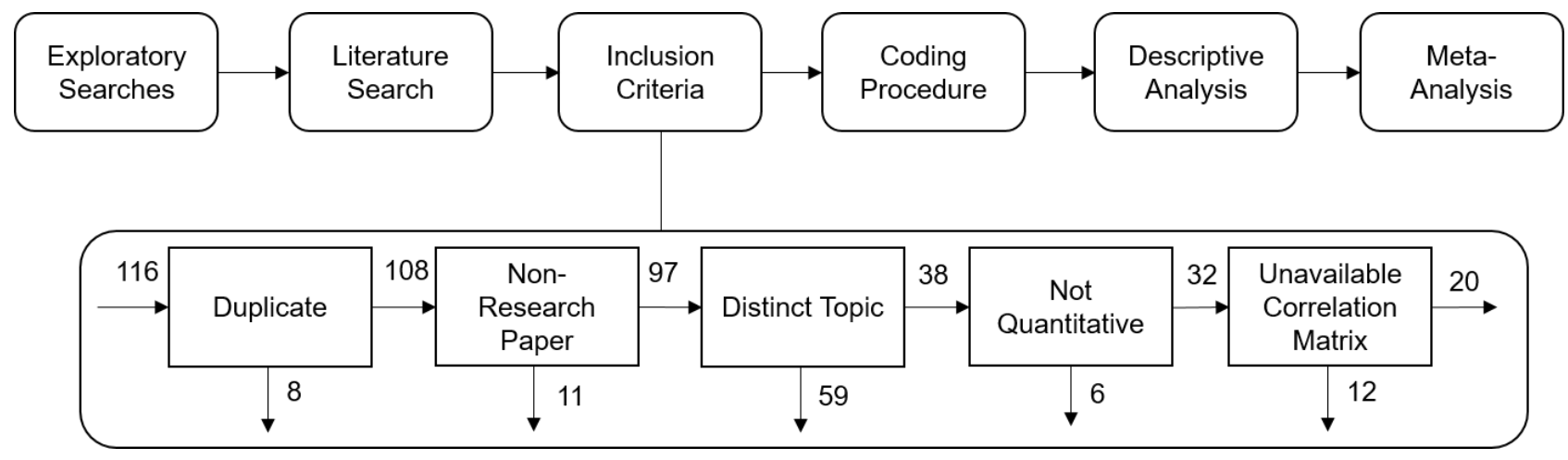

Figure 1: Analysis procedure flow diagram. Horizontal arrows represent remaining articles after each inclusion step whereas vertical arrows represent omitted articles.

\section{1. $\underline{\text { Search }}$}

Following guidelines of Webster and Watson (2002) and Ellis (2010), the analysis procedure commenced with a literature search. The search procedure was undertaken in the Scopus database (February 2015) which is the largest abstract and citation database of scholarly literature (Elsevier B.V 2014). Scopus is also the most relevant repository for studies within the disciplines where literature on why people adopt and use different technologies is being published. Among many others, Scopus also includes the AIS, ACM, IEEE and Science Direct libraries. 
We began the search process by conducting a set of exploratory searches of articles for discovering and identifying the common terminology in order to determine comprehensive but accurately describing set of keywords for formal search. This was first searching with rather wide terms while ordering the results by relevance and picking up some central keywords from clearly relevant studies and then making further searches with these revealed keywords. We also inspected studies that were referring already gathered relevant studies. Repeating this process iteratively while keeping accurate terms and discarding too wide keywords eventually resulted in acceptable search string. This basically consists of two parts: 1) action of making purchases and 2) context of virtual items and typical type of games or virtual worlds that allow such purchases. This search string was targeted for meta-data (titles, abstracts and keywords) of the articles rather than entire text and gave us 116 search hits. The complete search string is available in appendix A.

\subsection{Inclusion Criteria}

We determined 5 consecutive criteria for systematic and straightforward inclusion of studies for the analysis. The whole inclusion process resulted in 20 valid studies for further analysis by discarding 94 irrelevant or unsuitable articles from a total of 116 search hits.

First, the studies were inspected as to whether they were duplicates. Eight research articles were omitted for sharing same results or data with more recent and extended versions of these papers that were already included.

Second, 11 search hits were omitted since they were not full papers that had been published either in peer-reviewed journals, conferences or books.

Third and the largest omission category with 59 rejections was research articles that were on a different topic than the purchasing behavior of virtual items. In this category, most frequent topic 
was concerning interactive virtual product experience (e.g. inspecting virtual model of real item or wearing it on an avatar) and its effects on purchasing behavior of non-virtual items. This category also contained studies focusing on behavioral outcomes of augmented reality in purchasing decisions, effect of brand advertising in virtual worlds on real product purchase intentions and development reports of different virtual product systems in addition to less frequent various topics.

As a fourth step, the remaining entries were inspected for whether they included a quantitative empirical study. On this basis, 6 entries were omitted since the meta-analysis required actual measures from empirical studies with a similar research problem.

Finally, and most pertinently to the meta-analysis calculations, the studies had to report correlations between their variables. A total of 12 studies were omitted for not reporting a correlation matrix.

\subsection{Coding}

Before the actual analysis, the data is required to be in comparable format. When analyzed studies reported correlation matrixes, it did not require any extensive modifications in this study. However, five studies instead reported squared correlations which required square rooting in our data. While this was straightforward process, squared correlations should be interpreted with caution since possibly negative correlations gain positive sign when squared. In such cases, there is no other way to ensure that correlations are truly positive than trust that the authors are not hiding this information. Nevertheless, the sign of correlation between two variables can be reasoned with feasible accuracy and we trusted that the authors should report surprising negative correlations.

We identified three different types of variables related to measuring purchasing behavior of virtual items: intention to purchase, actual purchasing behavior and loyalty. Clear majority of the studies were interested in predicting general purchasing intention which was simply coded as "purchase 
intention”. Unfortunately, the research concerning loyalty and actual purchase behavior was so rare and scattered that we could not analyze these variables comprehensively in our meta-analysis. Therefore, this review was forced to focus on purchasing intention.

In addition, we inspected all independent variables of all studies on whether they actually measured what the variable name indicated. As an example many studies measured “enjoyment”, however, some studies measured the enjoyment of using the core service while others were interested in the enjoyment of using virtual goods and even some investigated the enjoyment of shopping activity itself. We combined or separated these cases accordingly.

The literature search revealed three studies that contained several subgroup analyses. Fortunately, these studies collected their subgroup questionnaire data separately from independent subject groups instead of splitting data afterwards. Therefore, all subgroup correlations could be treated as independent findings in this meta-analysis.

\subsection{Meta-analytic approach}

Reviewing published research can be divided into two overall approaches: 1) traditional qualitative method (also known as the narrative method) in which the conclusions of reviewed studies are practically summarized using words, and 2) meta-analysis which is a mathematical and quantitative approach, and where the effect sizes of the reviewed studies are combined using calculations (Ellis 2010). The narrative approach has been found to be insufficient when synthesizing findings from contradictory results, especially for a large number of studies (Hunter \& Schmidt 2004), whereas the meta-analytic approach provides more comprehensive results with estimates for effect size, different metrics for reliability, and information about different kinds of bias. Moreover, unlike the narrative approach, meta-analysis does not suffer from increased complexity in interpreting large amounts of studies. Instead, meta-analysis addresses the discrete limitations of individual studies 
and settles conflicting findings (Paré et al. 2015). As the limitations of traditional narrative review are acknowledged, it is reasonable to employ a meta-analysis in this particular study.

\subsubsection{Meta-analytic calculation model}

More specifically, meta-analysis is a mathematical and statistical method for combining the results of previous studies that address a similar research problem (or the data/results which can be used to address a similar research problem) (Glass 1981). There are two main approaches for mathematical meta-analysis (Hunter \& Schmidt 2004; Ellis 2010): one developed by Hunter and Schmidt (Hunter \& Schmidt 2000; Schmidt \& Hunter 1977) and the other by Hedges et al. (Hedges 1981; Hedges 1992; Hedges \& Olkin 1985; Hedges \& Vevea 1998). In the approach of Hedges et al., raw correlations are z-transformed before combining the effects, and weights of $n-3$ are used instead of the original sample size (n) for each study. In contrast, the method by Hunter and Schmidt uses untransformed correlations, and the original sample size of each study. However, an analysis using this approach should modify the weights to be taken into account and correct the study-specific faults such as measurement reliability. The calculation of Hedges et al.'s random effect model uses the between-studies variance (Ellis 2010). These two approaches will likely produce slightly different mean effect sizes and intervals, but it is difficult to say which one is better overall as the differences are minor (Ellis 2010). For example, Field (2005) ended up with results contradictory to a similar study of Hall \& Brannick (2002), even though both employed the two methods in similar conditions using Monte Carlo simulations. However, Johnson et al. (1995) compared extensively different meta-analytic approaches and concluded that the Hunter \& Schmidt method produces differing results and should be used with caution. Although Schmidt \& Hunter (1999) later argued that this difference was caused by use of an inappropriate formula for error correction, we were more confident with the method of Hedges et al. and it was chosen as an approach for the purposes of this analysis. 
Both meta-analytic calculation approaches include at least two different models, namely to account for fixed and random effects. In principle, a fixed effects model should be used when the studies share identical data collection conditions and a single value for the true effect is assumed. Thus, using a fixed effect generally produces less variance as well as tighter confidence intervals. On the other hand, a random effects model should be used when the study conditions are expected to vary, and the distribution for the true effect is assumed. Indeed, in most real life scenarios and metaanalyses, it would be absurd to assume that identical study conditions exist between studies. Moreover, as our data clearly suggests dissimilar conditions with varying variable details as well as different cultures and demographics amongst the respondents, it is reasonable to employ a random effects meta-analysis. Therefore we used the approach of Hedges et al., and a random effects model in our meta-analysis. The calculation formulas used in our meta-analysis are available in appendix B.

\subsubsection{Test of heterogeneity}

Despite the assumption that a random effect basis is preferred to a fixed effects model when combining the effect sizes of independent studies, we verified our model approach using tests for heterogeneity. The heterogeneity of our data was tested with Q-statistics and $\mathrm{I}^{2}$-values for every relationship that was analyzed in meta-analysis (these heterogeneity tests are available in appendix C). The Q-statistic (Cochran 1954) is the classical measure for heterogeneity while the $\mathrm{I}^{2}$-value represents the percent of the variance explained by the heterogeneity of the data, and the minimum of $0 \%$ indicates that all variability is instead due to sampling error within trials (Higgins \& Thompson 2002). All Q-estimates were statistically significant at $\mathrm{p}<0.01$ and each $\mathrm{I}^{2}$-value was above $80 \%$ (mostly above $90 \%$ ). Thus, the random effect model is seen as a proper approach for conducting this particular meta-analysis. 


\subsubsection{Effect interpretation and publication bias}

Correlation effect sizes were interpreted using Cohen’s (1988) small, medium and large thresholds, and therefore the three classes for interpreting effect sizes were:

- $\quad$ Small (S) for values between $0.10-0.30$

- $\quad$ Medium (M) for values between 0.30 - 0.50

- $\quad$ Large (L) for values between 0.50 - 1.00

To address the problem of publication bias, failsafe $\mathrm{N}$ was calculated for each of the analyzed relationships. The fundamental concept is to determine the number of additional studies with zero result needed to nullify an effect. There are two main approaches for such calculations (Long 2001): one method based on the sum of the Z scores (Rosenthal 1979) while the other uses effect sizes (Orwin 1983). The latter was used in this analysis since it provides more accurate results without the need for an interpretation of statistical significance testing (Long 2001). Additionally, the method collaborates well with the classes for effect sizes featured in this study. We used the smallthreshold as criterion value for fail-safe studies, and zero for the mean effect size of the fail-safe studies (Eq. 1).

$$
\text { Failsafe } N=\frac{k(r-0.1)}{0.1}
$$

Where $\mathrm{k}$ is the number of studies in the analysis, $\mathrm{r}$ is the mean effect size, and value 0.1 (the small effect size threshold) is the criterion value for failsafe studies. A higher failsafe $\mathrm{N}$ value implies a more reliable result in the aspect of publication bias. For interpretation of the value, the failsafe $\mathrm{N} /$ $\mathrm{k}$-ratio should exceed the threshold of 2.0, otherwise publication bias might pose a potential problem (Sabherwal et al. 2006). 


\subsubsection{Moderator analysis}

The purpose of our moderator analysis is to examine the difference in meta-analysis results between two different types of games. The difference between the two correlation estimates is examined by way of Q-test, which tests the homogeneity and significance of variance between groups (Borenstein et al. 2009). Similar to actual meta-analysis, the test also requires some decisions regarding the calculation model to be used. First, one must choose between a fixed or random effect model, depending on how the within group estimates are to be calculated. Similar to the main metaanalysis, we had no reason to believe that even studies within the same game categories would have such identical research conditions, that a fixed effect could be assumed. Therefore, the subgroup estimates are calculated using a random effects model. As a second issue, one must decide whether to assume true between-studies variance for both subgroups or to estimate separate variances. However, a relatively low number of studies within subgroups does not allow for the separate variances for each group to be estimated with any reasonable accuracy. On the other hand, we had no reason to assume different variances for these groups, so the same within studies estimate for variance is used for both subgroups.

\section{3. $\quad$ RESULTS}

\subsection{Details of the reviewed studies}

The inclusion process resulted in 20 research papers for further analysis and they are represented in Table 1 . When counting also different sub-studies, the total number of studies is 24 . The studies have been published between years 2008 and 2015. Majority of the studies are journal articles and the data contains only single conference paper. Sample sizes range from 38 to 2481 with a mean of 529 and standard deviation of 612. 
Table 1: Analyzed studies.

\begin{tabular}{|c|c|c|c|c|}
\hline Study & $\mathrm{n}$ & Service & Service Type & Venue \\
\hline Animesh et al. (2011) & 354 & Second Life & Virtual world & MIS Quarterly \\
\hline Cheon (2013) & 343 & Second Life & Virtual world & Information Technology Management \\
\hline Chou \& Kimsuwan (2013) & 335 & $\mathrm{n} / \mathrm{a}$ & F2P Online Games & J. of Internet Banking \& Commerce \\
\hline Guo \& Barnes (2012) & 253 & World of Warcraft & MMORPG & J. of Computer Information Systems \\
\hline Guo \& Barnes (2011) & 250 & Second Life & Virtual world & Information \& Management \\
\hline \multirow[t]{3}{*}{ Hamari (2015) } & 2156 & Habbo Hotel & Virtual world & Int. J. of Information Management \\
\hline & 398 & Multiple & FPS Online Game & \\
\hline & 237 & Multiple & Social Network Game & \\
\hline Han \& Windsor (2013) & 225 & MCP & Mobile Game & Int. J. of Mobile Communications \\
\hline Huang (2012) & 176 & Multiple & Social Network Game & Internet Research \\
\hline Kaburuan et al. (2009) & 38 & Multiple & ANY & 9th ICE-B \\
\hline Kim (2012) & 256 & Cyworld & Virtual world & Cyberpsychology, Behavior, \& Social Networking \\
\hline \multirow[t]{2}{*}{ Kim et al. (2012) } & 217 & Cyworld & Virtual world & Information Systems Research \\
\hline & 197 & Habbo Hotel & Virtual world & \\
\hline Kim et al. (2011) & 225 & Cyworld & Virtual world & Information \& Management \\
\hline Liu \& Shiue (2014) & 194 & Multiple & Social Network Game & Social Behavior \& Personality \\
\hline \multirow[t]{2}{*}{ Luo et al. (2011) } & 576 & World of Warcraft & MMORPG & The Service Industries Journal \\
\hline & 400 & Maple Story & MMORPG & \\
\hline Mäntymäki \& Salo (2013) & 1045 & Habbo Hotel & Virtual world & Int. J. of Information Management \\
\hline Mäntymäki \& Salo (2011) & 2481 & Habbo Hotel & Virtual world & Computers in Human Behavior \\
\hline Mäntymäki et al. (2014) & 1225 & Habbo Hotel & Virtual world & Digital Services \& Information Intelligence \\
\hline Park \& Lee (2011) & 327 & $\mathrm{n} / \mathrm{a}$ & F2P Online Game & Computers in Human Behavior \\
\hline Shin (2008) & 311 & Multiple & Virtual world & Interacting with Computers \\
\hline Wang \& Chang (2014) & 477 & $\mathrm{n} / \mathrm{a}$ & Online Game & Information Systems Frontiers \\
\hline
\end{tabular}

The frequencies of different environment types are shown in Table 2. Twelve studies have been conducted in the context of virtual worlds and 7 studies in the context of games (SNGs: 3, MMOs: 3, and Mobile games: 1). A single did not specify the context. 
Table 2: Frequencies of virtual environment types.

\begin{tabular}{lcc}
\hline Service Type & $\mathrm{k}$ \\
\hline Virtual worlds & 12 \\
Games & 7 \\
& Social Network Games & 3 \\
& Massively Multiplayer Online Role-Playing Game (MMORPG) & 3 \\
& Mobile games & 1 \\
Any & & 1 \\
\hline
\end{tabular}

Most of the studies did report their experiment concerning more than a single service or did not report actual titles of services (7). Among the reported titles, most of the studies did use Habbo Hotel (5), Second Life (3) or Cyworld (3) virtual worlds. Most frequent game title was World of Warcraft but with frequency of only 2. Rest of the reported titles were used in single studies (Table 3).

Table 3: Frequencies of virtual environment titles.

\begin{tabular}{llc}
\hline Service & Service Type & $\mathrm{k}$ \\
\hline Various services & any & 9 \\
Habbo Hotel & Virtual world & 5 \\
Second Life & Virtual world & 3 \\
Cyworld & Virtual world & 3 \\
World of Warcraft & MMORPG & 2 \\
Maple Story & MMORPG & 1 \\
The Mystery of the Crystal Portal & Mobile Game & 1 \\
\hline
\end{tabular}

We also investigated the distribution of theoretical frameworks utilized in the body of literature. As seen in Table 4, our review reveals that majority of the studies did not specify any clear theoretical 
foundation or used variety of variables from different frameworks or studies. Moreover, use of specific theories and models is rather scattered and only low frequencies are detected. Nevertheless, some studies utilized technology acceptance model (Davis et al. 1989), theory of planned behavior (Ajzen 1991) and unified theory of acceptance and use of technology (Venkatesh et al. 2003).

Table 4: Frequencies of theoretical backgrounds.

\begin{tabular}{lc}
\hline Theoretical Background & $\mathrm{k}$ \\
\hline Various & 7 \\
Technology Acceptance Model & 2 \\
Stimulus-Organism-Response Model & 2 \\
Transaction Cost Theory + Unified Theory of Acceptance and Use of Technology & 2 \\
Theory of Planned Behavior & 1 \\
Expectancy Disconfirmation Model & 1 \\
Unified Theory of Acceptance and Use of Technology & 1 \\
Theory of Consumption Values & 1 \\
Virtual Experiential Marketing & 1 \\
Customer Value Theory & 1 \\
Self-Presentation Theory & 1
\end{tabular}

\subsection{Variables}

In total, the collected data contains large number of different variables and 398 unique correlation pairs. As we were interested in factors that explain virtual goods purchases, we meta-analyzed the correlations between the purchase-related variable and any variables that were featured in at least 3 individual studies. Table 5 introduces the most frequent variables in the reviewed research 
literature, number of studies examining them $(\mathrm{k})$ as well as a brief description for each variable. These variables are also featured in meta-analysis. 
Table 5: Most frequent variables and their brief descriptions.

\begin{tabular}{|c|c|c|}
\hline Variable & $\mathrm{k}$ & Description \\
\hline $\begin{array}{l}\text { Purchase } \\
\text { Intention }\end{array}$ & 24 & Intention to purchase virtual goods (Ajzen \& Fishbein 1980). \\
\hline $\begin{array}{l}\text { Service Use } \\
\text { Enjoyment }\end{array}$ & 8 & Extent of how enjoyable and fun using the game or virtual world itself is. \\
\hline $\begin{array}{l}\text { Subjective } \\
\text { Norms }\end{array}$ & 8 & $\begin{array}{l}\text { Perceived social pressure from other people on how acceptable playing games or } \\
\text { using virtual worlds is (Ajzen \& Fishbein 1980). Also often referred as "social } \\
\text { norms" or "social influence". }\end{array}$ \\
\hline Flow & 6 & $\begin{array}{l}\text { Flow is a mental state where a person is fully immersed, deeply concentrated and } \\
\text { truly enjoys when performing a certain activity (Csíkszentmihályi 1990). Flow is } \\
\text { the optimal hedonic experience in playing games or using virtual worlds. }\end{array}$ \\
\hline $\begin{array}{l}\text { Attitude Toward } \\
\text { Purchase }\end{array}$ & 5 & $\begin{array}{l}\text { Attitude is own opinion on how positive or negative purchasing virtual goods is } \\
\text { (Ajzen \& Fishbein 1980). }\end{array}$ \\
\hline $\begin{array}{l}\text { Service Use } \\
\text { Intention }\end{array}$ & 4 & Intention to play games or use virtual worlds (Ajzen \& Fishbein 1980). \\
\hline $\begin{array}{l}\text { Perceived Ease } \\
\text { of Use }\end{array}$ & 3 & $\begin{array}{l}\text { "The degree to which an individual believes that using a particular system would } \\
\text { be free of physical and mental effort" (Davis 1989). Especially in context of games, } \\
\text { ease of use denotes effortless in user interface rather than difficulty level. }\end{array}$ \\
\hline Perceived & 3 & Perception on amount of friends, peers and people around are also using the \\
\hline Network Size & & service. \\
\hline Perceived Value & 3 & $\begin{array}{l}\text { Perceived ratio between value and price or virtual goods in which valuable but } \\
\text { cheap goods become desirable whereas expensive items make users consider } \\
\text { alternatives. }\end{array}$ \\
\hline Self- & 3 & Desire for expressing oneself in virtual world by character customization such as \\
\hline Presentation & & wearing stylish clothing and accessories on avatar. \\
\hline Social Presence & 3 & Sense of real human contact and sociability in virtual world. \\
\hline
\end{tabular}

$k=$ number of studies examining the variable. 


\subsection{Meta-analysis}

\subsubsection{Main findings}

The results in Table 6 (also visualized in Figure 2) show most frequently studied variables in the literature and our meta-analytically produced estimates for their correlation with Purchase Intention. The strongest predictor for virtual good purchases was Attitude $\left(0.662^{* * *}\right)$ which can be classified as having a large effect. In addition, the meta-analysis found nine significant and medium-sized effects: Flow $\left(0.482^{* * *}\right)$, Perceived Network Size $(0.480 * * *)$, Self-Presentation $(0.478 * * *)$ and Subjective Norms $(0.466 * * *)$ had rather strong correlations with Purchasing Intention. Moreover, effects of Social Presence $(0.438 * * *)$ and Perceived Value $(0.418 * * *)$ represented middle ground of medium sized effects in the analysis. Finally, Service Use Enjoyment $\left(0.370^{* * *}\right)$, Service Use Intention $\left(0.359^{* * *}\right)$, and Perceived Ease of Use $\left(0.333^{* * *}\right)$ showed weakest correlation estimates with Purchasing Intention. Nevertheless, every estimate in the analysis was clearly positive and above medium effect size threshold as well as statistically significant at $\mathrm{p}<0.001$ with adequate failsafe $\mathrm{N}$ values. 
Table 6: Results of the meta-analysis. $k=$ number of studies, $\Sigma n=$ cumulative sample size, $C=$ correlation class, $r=$ correlation coefficient, lower and higher bounds of $95 \%$ confidence interval, $Z=z$-score for correlation estimate, $p=$ statistical significance of estimate, fs $N=$ failsafe $N$. Significance levels: ${ }^{* \star} p<0.001$, ${ }^{\star \star} p<0.01,{ }^{*} p<0.05,{ }^{n s} p>0.05$

\begin{tabular}{|c|c|c|c|c|c|c|c|c|c|c|}
\hline \multirow[b]{2}{*}{ Variables } & & \multirow[b]{2}{*}{ k } & \multirow[b]{2}{*}{$\sum n$} & \multirow[b]{2}{*}{ C } & \multirow[b]{2}{*}{ r } & \multicolumn{2}{|c|}{$95 \%$ Conf. Int. } & \multirow[b]{2}{*}{ Z } & \multirow[b]{2}{*}{$\mathrm{p}$} & \multirow[b]{2}{*}{ fs $N$} \\
\hline & & & & & & low & high & & & \\
\hline Service Use Enjoyment & x Purchase Intention & 8 & 8045 & $M$ & $0.370^{\star \star \star}$ & 0.275 & 0.459 & 7.144 & 0.000 & 22 \\
\hline Subjective Norms & x Purchase Intention & 8 & 3868 & M & $0.466^{\star \star \star}$ & 0.294 & 0.608 & 4.915 & 0.000 & 30 \\
\hline Flow & x Purchase Intention & 6 & 2043 & M & $0.482^{\star \star \star}$ & 0.399 & 0.557 & 10.015 & 0.000 & 23 \\
\hline Attitude Toward Purchasing & x Purchase Intention & 5 & 3102 & L & $0.662^{\star \star \star}$ & 0.597 & 0.719 & 14.405 & 0.000 & 29 \\
\hline Service Use Intention & x Purchase Intention & 4 & 5272 & M & $0.359 * \star \star$ & 0.247 & 0.461 & 5.966 & 0.000 & 11 \\
\hline Perceived Ease of Use & x Purchase Intention & 3 & 3837 & M & $0.333^{\star \star \star}$ & 0.216 & 0.440 & 5.360 & 0.000 & 7 \\
\hline Perceived Network Size & x Purchase Intention & 3 & 4751 & M & $0.480 * \star \star$ & 0.401 & 0.551 & 10.469 & 0.000 & 12 \\
\hline Perceived Value & x Purchase Intention & 3 & 759 & M & $0.418^{\star \star \star}$ & 0.331 & 0.497 & 8.637 & 0.000 & 10 \\
\hline Self-Presentation & x Purchase Intention & 3 & 639 & M & $0.478^{\star \star \star}$ & 0.296 & 0.626 & 4.743 & 0.000 & 12 \\
\hline Social Presence & x Purchase Intention & 3 & 2624 & M & $0.438^{\star \star \star *}$ & 0.333 & 0.532 & 7.444 & 0.000 & 11 \\
\hline
\end{tabular}




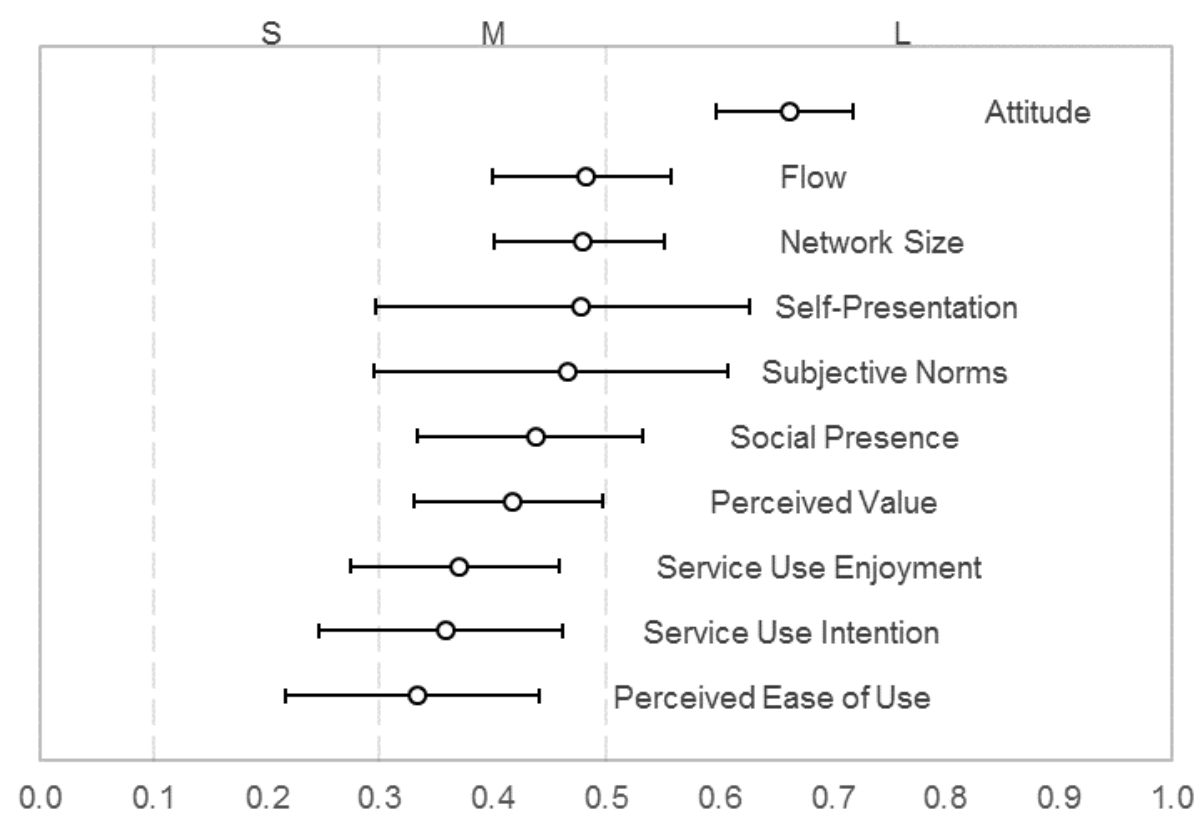

Figure 2: Meta-analysis of correlations with purchase intention and their $95 \%$ confidence intervals. $S, M, L=$ small, medium and large classes for correlation strength.

\subsubsection{Moderating effect of service type}

While games and virtual worlds offer purchasable virtual goods, they are relatively different types of environments. Whereas games are commonly competitive, rule-driven, fast-paced goal-orientated and narrative rich, virtual worlds are commonly free-form and have no clearly defined goals or game-like competition. In games, purchasing virtual goods can give unfair competitive advantage as they can make the game character stronger (Lehdonvirta 2009; Hamari \& Lehdonvirta 2010; Hamari 2015; Alha et al. 2014). Therefore, the motivations for purchasing virtual goods in these environments may differ. To address this assumption, we expanded the meta-analysis by investigating the differences between effect between the game and virtual world environments.

Since the number of studies become lowered due to the grouping, we reduced the required $k$ of studies to two for each category. As a result, the comparison analysis compares five relationships 
between the virtual environment categories (Table 7 and Figure 3). The results showed a large difference for correlation between Service Use Intention and Purchase Intention $\left(\mathrm{Q}=46.651^{* * *}\right)$, where games had considerably lower correlation $\left(0.211^{* * *}\right)$ compared to mediocre estimate of virtual worlds (0.465***). Quite similarly, there was a large difference in correlation between Service Use Enjoyment and Purchase Intention $(\mathrm{Q}=22.492 * * *)$, where again the relationship for games $\left(0.185^{* * *}\right)$ was significantly weaker than the estimate for virtual worlds $\left(0.461^{* * *}\right)$. Moreover, there was slight difference between correlations for Flow and Purchase Intention $(\mathrm{Q}=$ $5.920 *)$, where games had a lower estimate $(0.437 * * *)$ compared to virtual worlds $(0.557 * * *)$. However, the analysis could not detect significant difference for correlation between Subjective Norms and Purchase Intention $\left(\mathrm{Q}=0.052^{\mathrm{ns}}\right)$ since both categories had similar estimates (games: $0.453^{* * *}$, worlds: $\left.0.494^{* * *}\right)$. In addition, there was no noticeable difference in relationship between Attitude and Purchase Intention $\left(\mathrm{Q}=0.027^{\mathrm{ns}}\right)$ as both categories showed similarly high correlations (games: $0.666^{* * *}$, worlds: $0.654^{* * *}$ ). 
Table 7: Differences between game and non-game environments. $k=$ number of studies, $\sum n=$ cumulative sample size, $C=$ correlation class, $r=$ correlation coefficient, lower and higher bounds of $95 \%$ confidence interval, $Z=z$-score for correlation estimate, $p=$ statistical significance of estimate, $f \mathrm{~s} N=$ failsafe $N$. Significance levels: ${ }^{* *} p<0.001,{ }^{* *} p<0.01,{ }^{*} p<0.05,{ }^{n s} p>0.05, Q=Q$ test value, $P=$ significance of $Q$ test value

\begin{tabular}{|c|c|c|c|c|c|c|c|c|c|c|}
\hline \multirow[b]{2}{*}{ Variables } & \multirow[b]{2}{*}{ k } & \multirow[b]{2}{*}{$\sum \mathrm{n}$} & \multirow[b]{2}{*}{$\mathrm{C}$} & \multirow[b]{2}{*}{$r$} & \multicolumn{2}{|c|}{$95 \%$ Conf. Int. } & \multirow[b]{2}{*}{ Z } & \multirow[b]{2}{*}{$\mathrm{p}$} & \multicolumn{2}{|c|}{ Q difference test } \\
\hline & & & & & low & high & & & $\mathrm{Q}$ & $P$ \\
\hline \multicolumn{11}{|c|}{ Service Use Intention x Purchase Intention } \\
\hline Games & 2 & 635 & S & $0.211^{\star \star \star}$ & 0.135 & 0.284 & 5.367 & 0.000 & $46.651^{\star \star \star}$ & 0.000 \\
\hline Worlds & 2 & 4637 & M & $0.465^{\star \star \star}$ & 0.443 & 0.488 & 34.314 & 0.000 & & \\
\hline
\end{tabular}

Service Use Enjoyment $x$ Purchase Intention

$\begin{array}{lllllllllll}\text { Games } & 3 & 888 & \mathrm{~S} & 0.185^{\star \star \star} & 0.101 & 0.268 & 4.246 & 0.000 & 22.492^{\star * *} & 0.000 \\ \text { Worlds } & 5 & 7157 & \mathrm{M} & 0.461^{\star \star *} & 0.383 & 0.532 & 10.304 & 0.000 & & \end{array}$

Flow x Purchase Intention

$\begin{array}{lllllllllll}\text { Games } & 4 & 1346 & \mathrm{M} & 0.437^{\star \star *} & 0.347 & 0.519 & 8.653 & 0.000 & 5.920^{\star} & 0.015 \\ \text { Worlds } & 2 & 697 & \mathrm{~L} & 0.557^{\star \star \star} & 0.504 & 0.607 & 16.540 & 0.000 & & \end{array}$

Subjective Norms x Purchase Intention

$\begin{array}{lllllllllll}\text { Games } & 4 & 1113 & M & 0.453^{\star \star \star} & 0.209 & 0.644 & 3.461 & 0.001 & 0.052^{\text {ns }} & 0.820 \\ \text { Worlds } & 3 & 2717 & M & 0.494^{\star \star} & 0.179 & 0.717 & 2.940 & 0.003 & & \end{array}$

Attitude Toward Purchasing $x$ Purchase Intention

\begin{tabular}{lllllllllll} 
Games & 2 & 635 & $\mathrm{~L}$ & $0.666^{\star \star \star}$ & 0.587 & 0.733 & 12.042 & 0.000 & $0.027^{\text {ns }}$ & 0.868 \\
Worlds & 2 & 2467 & $\mathrm{~L}$ & $0.654^{\star \star \star}$ & 0.515 & 0.760 & 7.185 & 0.000 & & \\
& & & & & & & & & & \\
\hline
\end{tabular}

Despite the fact that number of studies is lowered to two studies at minimum, all group estimates are statistically significant and positive. However, in relationship between Subjective Norms and Purchase Intention, correlation estimates for both service categories had wide confidence intervals (see Figure 3) due to high variation in previous research findings. Games-group had $95 \%$ confidence interval of 0.435 whereas virtual world-category had 0.539 in difference between the 
interval bounds. Although the literature showed rather varying findings on strength of the relationship between the variables, the correlation estimates in this analysis were clearly positive in both categories. Other studies within their categories had rather unanimous results which is shown in relatively narrow confidence intervals (0.245 at most).

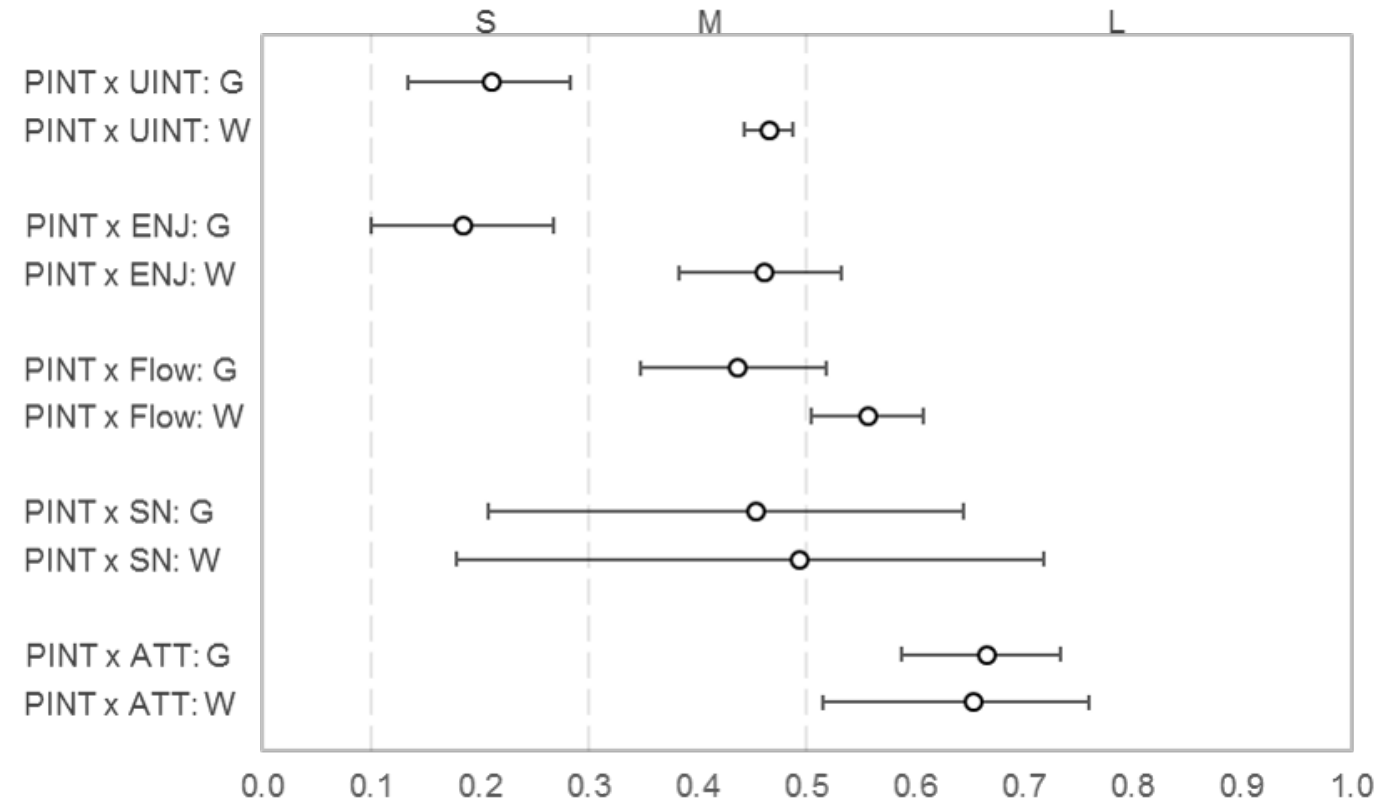

Figure 3: Difference in correlations with purchase intention between games and virtual worlds. PINT = purchase intention, UINT = core service use intention, $S N=$ subjective norms, $A T T=$ attitude toward purchasing virtual goods, ENJ = core service use enjoyment, $G=$ Games, $W=$ Virtual worlds. $S, M, L=$ small, medium and large correlation classes.

\section{DISCUSSION}

This study investigated the question of why do people purchase virtual goods by conducting a metaanalysis of the existing quantitative body of literature. The results revealed that across the literature the following factors were most strongly associated with purchase behavior for virtual goods: attitude, flow, network size, self-presentation needs, subjective norms, social presence, perceived value, service use enjoyment, service use intention and perceived ease of use. Attitude towards 
virtual goods had clearly the strongest association with purchase intention. Moreover, the results showed differences in the magnitude of some purchase motivators between games and virtual worlds: in virtual worlds, service use intention and enjoyment were significantly stronger predictors for virtual good purchases than in games.

In contrast to consumer research in general, as can be seen from the set of variables examined in the literature, the research on virtual goods consumption has rather heavily focused on aspects related to the platform on which the virtual goods are being used, whereas literature on consumption of goods in general is commonly focused on the aspects of the products themselves. The findings of this study and the focus in the literature strongly indicate that virtual goods are being consumed in a context that is heavily tied to the value formation of virtual goods. Virtual goods inhabit a highly curious environment: virtual goods are bound by the rules of the service in which they are used, developers control the supply and value of virtual goods by controlling how new virtual goods can be spawned into existence, how they can be traded, who can own them at any particular time, their price, their rate of degradation and whether they can be traded back to 'real money'. Ultimately, any of this does not matter unless the developers have also created an appealing and enjoyable enough environment to which users are attached to. Without users using the platform, the virtual goods within remain in a limbo of virtual meaning. Customers do not choose the games they start playing based on what purchasable goods the game might have, and therefore, it creating an appealing platform that hosts the virtual goods remain an important prerequisite. Instead, users are arguably more likely to choose the core services based on their entertainment value or interestingness. Thus, in order to enable purchases, potential customers must first use the core service and enjoy it as such. Indeed, it is also the result of this study that confirm that user enjoyment, flow, healthy community around the platform, and intentions to continue using the platform are important factors for virtual good purchases (and their value) (See. E.g. Fairfield 2005; Lehdonvirta 2009; Hamari \& Lehdonvirta 2010; Lehdonvirta \& Castronova 2014). Therefore, even though actual products that 
generate revenue are the sold virtual goods, practitioners should ensure that the core service is enjoyable, interesting and of high quality on its own. The real challenge then is to create incentives for purchasing virtual goods without compromising the user experience (Alha et al. 2014; Hamari \& Lehdonvirta 2010; Hamari 2015; Lehdonvirta \& Castronova 2014; Lin \& Sun 2011).

In relation to the enjoyment derived from using the platform, our results surprisingly show that, enjoyment had a smaller impact on purchase behavior in games (small effect) that in virtual worlds (medium effect). At first, this finding might seem unintuitive since games, after all, are commonly strongly associated with the pursuit of enjoyment. However, recent related literature may shed light on possible explanations for this results. Prior literature examining the association between purchase behavior of virtual goods in games and the game experience (Hamari 2015; Lin \& Sun 2011) has observed and discussed that the enjoyment (and related factors) may have a more complex, dual-directional effect on purchases in games. In order to create demand for the virtual goods in games, many game developers may intentionally seek to frustrate players by creating artificial obstacles and hindrances, and therefore, generate sales through negative enjoyment' (Hamari \& Lehdonvirta 2010; Hamari 2015; Lin \& Sun 2011). Therefore, on one hand, developers are required to make the game enjoyable enough for the players to come and stay in the game, but on the other hand, it may be in the developer's best interest to then frustrate the players in order to sell them more virtual goods that address those frustrations. Indeed, our results may lend support for these prior findings; the low effect size between enjoyment and purchase intentions in games may suggest that there is a double-sided effect: on one hand, enjoyment by default increase willingness to purchase virtual goods (especially through increased playing intentions) but on the other hand, virtual goods are purchased if the game is not enjoyable enough. Relatedly, the strength of association between playing and purchase intentions also varied between games and virtual worlds in the same manner as the relationship between enjoyment and playing intentions: in virtual worlds 
the association was of medium strength whereas in games it the association was only of small strength.

The results show a strong positive relationship between attitude and virtual good purchases independent of service type. This relationship might be more crucial in games, which can be regarded as the more competitive setting than virtual worlds. Many free-to-play game developers utilize the competitive nature of games and offer virtual goods that have functional advantages such as more powerful weapons, better armors or items to temporary improve character statistics. Although the business strategy is currently widely used, many players are uncomfortable with paying for unfair performance boost (Lin \& Sun 2007; 2011). It remains an interesting further line of inquiry to investigate what factors may alleviate or strengthen these strong attitudes towards virtual good purchases.

Subjective norm is central variable in theory of planned behavior (Ajzen 1991). According to this theory, human behavior is affected by social pressure on whether such behavior is accepted or not by others. Beneficial to the theory, our meta-analysis results confirm that purchase intention has significant correlation with subjective norms, indicating that when purchasing virtual goods is accepted by others, people themselves are more likely to make purchases. While the results of this study certainly confirm an effect, they also show a large variation between the studies. However this high variance could be explained with individual differences in accepting social influence. Some people might perceive opinions of other more important whereas some might perform actions without particularly considering what others think of them. Alternatively, some platforms may lack features that would facilitate social interaction, and therefore, also the level of social influence may be diminished. Despite the possible high variation in individuals, opinions of others definitely have an effect on own purchase motivation. 
Flow has been characterized as an optimal hedonic experience in use of service in which the user is deeply concentrated and fully immersed in the activity (Csíkszentmihályi 1990). As flow represents another hedonic experience in use of service, it is arguably related to enjoyment. However, the key difference between the variables is that flow can be regarded as more specific experience whereas enjoyment represents more general enjoyment in service use. Thus, we expected finding stronger association between flow and purchase intention than enjoyment had. Our meta-analysis revealed significant and relatively strong correlation between flow and purchase intention. Moreover, our results showed noticeably stronger association between flow and purchase intention than enjoyment had.

In technology acceptance model (Davis 1989), perceived ease of use predicts the use intention of information systems. However, this analysis examined its relationship with purchase intention. Although direct effect of ease of use was not modeled by any of the analyzed studies, we were able to estimate its correlation with purchase intention. Our meta-analysis results showed significant relationship between the variables, indicating that higher core service usability can increase purchase intentions. However, the correlation was only mediocre in its strength and the weakest relationship in this meta-analysis. On the other hand, we believe that ease of use would have more significant role by indirectly affecting purchase intention. Thus, future meta-analyses could examine the relationship between ease of use and other variables. Moreover, they could employ structural equation modeling approach and examine the indirect relationship of ease of use and purchase intention through other variables.

\subsection{Limitations}

There are some limitations in this review that should be acknowledged. First, although metaanalysis can be technically conducted when a relationship has been examined by at least two 
studies, most analyses typically require more findings. Due to relatively low number of previous quantitative research and especially because of scattered nature of research literature, this analysis occasionally contained rather low number of studies for some of the correlation pairs. While we managed to achieve significant relationships and mostly narrow confidence intervals for our estimates, some correlation pairs would preferably need more independent findings for wider generalization. In any case, however, the current meta-analysis offers the most reliable, accurate and generalizable results up to date on the topic by synthesizing results from several high quality and full sized studies.

As discussed, virtual goods can be divided into two categories by their characteristics. "Functional" goods are items that improve performance or progression in the service such as more powerful weapons, unlockable new content and temporary improvements to virtual character. On the other hand, “appearance”-based goods do not grant any functional benefits and instead only alter the look of the virtual character. Since there is noticeable difference between these two types of goods, the motivations for purchasing can also be expected to vary. However, only a few studies clearly reported the type of virtual goods, preventing us from analyzing differences between the attractiveness of functional and appearance based virtual goods. Future efforts could examine the motivational differences between purchasing the two types of virtual goods.

Since we aimed for as comprehensive as possible literature review of quantitative research and meta-analysis, we were not restricted by theoretical assumptions stemming from theoretical frameworks such as Technology Acceptance Model (Davis 1989) or Theory of Planned Behavior (Ajzen 1991). We examined the correlations between variables regardless of whether the analyzed studies had modelled a relationship between them in their structural models. Therefore, our study not only presents reliable results on the topic why people purchase virtual goods but is also able to take into account relationships between variables not disclosed as results in prior literature. Although using zero-order correlations provides the most accurate and unbiased estimates as in this 
review, it is not uncommon for meta-analyses to instead use standardized regression path coefficients as their metric. Future meta-analyses could respectively synthesize previous path coefficients, however, it is important to note that model structure significantly affects these estimates. Thus, such meta-analyses should not interpret relationship strengths too strictly and perhaps instead examine proportion of significant effects. In either case, using correlations provides the most reliable estimates for relationships between variables.

Finally, we encourage structural equation modeling studies to report correlation matrixes not only to allow their data to be included in future correlation meta-analyses but also to address convergent and discriminant validity. Although majority of the reviewed studies followed this practice, unfortunately many did address only other of the validities or showed no results for neither of these potential issues. We also would like to emphasize scholars not to hide measures such as nonsignificant path coefficients and total variance in dependent variable explained by independent variables $\left(\mathrm{R}^{2}\right)$ in the research model. Unreported non-significant path coefficients are especially unfortunate from the view point of meta-analyses of path coefficients, but also not reporting the $\mathrm{R}^{2}$ values conceals the predictive performance in the model. 


\section{REFERENCES}

Ajzen, I. (1991) The theory of planned behavior, Organizational behavior and human decision processes 50(2): 179-211.

Ajzen, I. and Fishbein, M. (1980) Understanding Attitudes and Predicting Social Behavior, Englewood Cliffs, NJ: Prentice-Hall.

Alha, K., Koskinen, E., Paavilainen, J., Hamari, J. and Kinnunen, J. (2014) Free-to-Play Games: Professionals’ Perspectives, Proceedings of Nordic Digra 2014, Gotland, Sweden, May 29.

Animesh, A., Pinsonneault, A., Yang, S.-B. and Oh, W. (2011) An odyssey into virtual worlds: Exploring the impacts of technological and spatial environments on intention to purchase virtual products, MIS Quarterly 35(3): 789-810.

Bartle, R. (2004) Pitfalls of virtual property, Themis Group.

Borenstein, M., Hedges, L. V. Higgins, J. P. T. and Rothstein, H. R. (2009) Introduction to metaanalysis, Chichester: Wiley.

Cha, J. (2011) Exploring the internet as a unique shopping channel to sell both real and virtual items: A comparison of factors affecting purchase intention and consumer characteristics, Journal of Electronic Commerce Research 12(2): 115-132.

Cheon, E. (2013) Energizing business transactions in virtual worlds: An empirical study of consumers' purchasing behaviors, Information Technology and Management 14(4): 315-330.

Chou, C.-M. and Kimsuwan, A. (2013) Factors affecting purchase intention of online game prepayment card - Evidence from Thailand, Journal of Internet Banking and Commerce 18(3): 113. 
Cochran, W. G. (1954) The combination of estimates from different experiments, Biometrics 10(1): 101-129.

Cohen, J. (1988) Statistical Power Analysis for the Behavioral Sciences, 2nd ed. Hillsdale: Lawrence Erlbaum.

Csíkszentmihályi, M. (1990) Flow: The psychology of optimal experience, New York, NY: Harper and Row.

Davis, F. D. (1989) Perceived usefulness, perceived ease of use, and user acceptance of information technology, MIS Quarterly 13(3): 319-340.

Davis, F. D., Bagozzi, R. P. and Warshaw, P. R. (1989) User acceptance of computer technology: a comparison of two theoretical models, Management Science 35(8): 982-1003.

Domina, T., Lee, S.-E. and MacGillivray, M. (2012) Understanding factors affecting consumer intention to shop in a virtual world, Journal of Retailing and Consumer Services 19(6): 613-620.

Ellis, P. D. (2010) The essential guide to effect sizes: Statistical power, meta-analysis, and the interpretation of research results, Cambridge: Cambridge University Press.

Elsevier B.V, (2014) Online tools - Scopus, [Online]

Available at: http://www.elsevier.com/online-tools/scopus

[Accessed 2410 2014].

Fairfield, J. (2005) Virtual property, Boston University Law Review 85: 1047.

Field, A. P. (2005) Is the meta-analysis of correlation coefficients accurate when population correlations vary?, Psychological Methods 10(4): 444-467. 
Forbes, (2013) Social Network Games Shed 10 Million Players...in One Month, [Online]

Available at: http://www.forbes.com/sites/carolpinchefsky/2013/04/17/social-network-games-shed10-million-players-in-one-month/

[Accessed 23 November 2014].

Gao, J. (2014) An empirical research on consumers' purchasing behavior of virtual products in SNS, Research Journal of Applied Sciences, Engineering and Technology 7(7): 1279-1286.

Glass, G. V. (1981) Meta-Analysis in Social Research, Beverly Hills, CA: Sage Publications.

Goel, L., Johnson, N. A. Junglas, I. and Blake, I. (2011) From space to place: Predicting users' intention to return to virtual worlds, MIS Quarterly 35(3): 749-771.

Guo, Y. and Barnes, S. (2011) Purchase behavior in virtual worlds: An emprical investigation in Second Life, Information \& Management 48(7): 303-312.

Guo, Y. and Barnes, S. (2012) Explaining purchasing behavior within World of Warcraft, Journal of Computer Information Systems 52(3): 18-30.

Hall, S. M. and Brannick, M. T. (2002) Comparison of two random-effects methods of metaanalysis, Journal of Applied Psychology 87(2): 377-389.

Hamari, J. (2011) Perspectives from behavioral economics to analyzing game design patterns: Loss aversion in social games, Vancouver, Canada, May 7-12. CHI'2011 (Social games workshop).

Hamari, J. (2015) Why do people buy virtual goods? Attitude toward virtual good purchases versus game enjoyment, International Journal of Information Management 35(3): 299-308. 
Hamari, J. and Järvinen, A. (2011) Building Customer Relationship through Game Mechanics in Social Games, in M. Cruz-Cunha, V. Carvalho \& P. Tavares, (ed.) Building Customer Relationship through Game Mechanics in Social Games, Hershey, PA: IGI Global, pp. 348-365.

Hamari, J. and Keronen, L. (2016) Why do people buy virtual goods? A literature review, 49th Hawaii International Conference on System Sciences (HICSS) 1358-1367.

Hamari, J. and Lehdonvirta, V. (2010) Game Design as Marketing: How Game Mechanics Create Demand for Virtual Goods, International Journal of Business Science \& Applied Management 5(1): 14-29.

Hamari, J. and Tuunanen, J. (2014) Player types: A meta-synthesis, Transactions of the Digital Games Research Association 1(2): 29-53.

Han, B. and Windsor, J. (2013) An investigation of the smartphone user's in-game purchase intention, International Journal of Mobile Communications 11(6): 617-635.

Harviainen, J. T. and Hamari, J. (2015) Seek, share, or withhold: Information trading in MMORPGs, Journal of Documentation 71(6).

Hedges, L. V. (1981) Distribution theory for Glass's estimator of effect size and related estimators, Journal of Educational Statistics 6(2): 106-128.

Hedges, L. V. (1992) Meta-analysis, Journal of Educational Statistics 17(4): 279-296.

Hedges, L. V. and Olkin, I. (1985) Statistical Methods for Meta-Analysis, London: Academic Press.

Hedges, L. V. and Vevea, J. L. (1998) Fixed- and random-effects models in meta-analysis, Psychological Methods 3(4): 486-504. 
Higgins, J. P. T. and Thompson, S. G. (2002) Quantifying heterogeneity in a meta-analysis, Statistics in medicine 21(11): 1539-1558.

Huang, E. (2012) Online experiences and virtual goods purchase intention, Internet Research 22(3): 252-274.

Hunter, J. E. and Schmidt, F. L. (2000) Fixed effects vs. random effects meta-analysis models: Implications for cumulative research knowledge, International Journal of Selection and Assessment 8(4): 275-292.

Hunter, J. E. and Schmidt, F. L. (2004) Methods of Meta-Analysis: Correcting Error and Bias in Research Findings, 2nd ed. Newbury Park, CA: Sage Publications.

Johnson, B. T. Mullen, B. and Salas, E. (1995) Comparison of three major meta-analytic approaches, Journal of Applied Psychology 80(1): 94-106.

Kaburuan, E. R. Chen, C.-H. and Jeng, T.-S. (2009) Identifying users' behavior purchasing virtual items, The 9th International Conference on Electronic Business, Macau, November 30 - December 4, 2009 250-256.

Kim, B. (2012) Understanding key factors of users' intentions to repurchase and recommend digital items in social virtual worlds, Cyberpsychology, Behavior, and Social Networking 15(10): 543-550.

Kim, H.-W., Chan, H. C. and Kankahalli, A. (2012) What motivates people to purchase digital items on virtual community websites? The desire for online self-presentation, Information Systems Research 23(4): 1232-1245.

Kim, H.-W., Gupta, S. and Koh, J. (2011) Investigating the intention to purchase digital items in social networking communities: A customer value perspective, Information \& Management 48(6): 228-234. 
Kimppa, K. K. Heimo, O. I. and Harviainen, T. J. (2016) First dose is always freemium, ACM SIGCAS Computers and Society - Special Issue on Ethicomp 45(3): 132-137.

Lehdonvirta, V. (2009) Virtual item sales as a revenue model: Identifying attributes that drive purchase decisions, Electronic Commerce Research 9(1-2): 97-113.

Lehdonvirta, V. and Castronova, E. (2014) Virtual economies: Design and analysis, Cambridge, Massachusetts: MIT Press.

Lin, H. and Sun, C.-T. (2007) Cash trade within the magic circle: Free-to-play game challenges and massively multiplayer online game player responses, Proceedings of DiGRA 2007.

Lin, H. and Sun, T.-C. (2011) Cash trade in free-to-play online games, Games and Culture 6(3): 270-287.

Liu, H.-J. and Shiue, Y.-C. (2014) Influence of facebook game players' behavior on flow and purchase intention, Social Behavior and Personality 42(1): 125-134.

Long, J. (2001) An Introduction to and Generalization of the "Fail-Safe N", Paper presented at the Annual Meeting of the Southwest Educational Research Association (New Orleans, LA, February 13, 2001).

Luo, M. M. Chen, J.-S., Ching, R. K. and Liu, C.-C. (2011) An examination of the effects of virtual experiential marketing on online customer intentions and loyalty, The Service Industries Journal 31(13): 2163-2191.

Mäntymäki, M., Merikivi, J. and Islam, N. A. (2014) Young people purchasing virtual goods in virtual worlds: The role of user experience and social context, Digital Services and Information Intelligence 445: 303-314. 
Mäntymäki, M. and Salo, J. (2011) Teenagers in social virtual worlds: Continuous use and purchasing behavior in Habbo Hotel, Computers in Human Behavior 27(6): 2088-2097.

Mäntymäki, M. and Salo, J. (2013) Purchasing behavior in social virtual worlds: An examination of Habbo Hotel, International Journal of Information Management 33(2): 282-290.

Martocchio, J. J. and Webster, J. (1992) Effects of feedback and cognitive playfulness on performance in microcomputer software training, Personnel Psychology 45(3): 553-578.

Nieborg, D. B. (2015) Crushing candy: The free-to-play game in its connective commodity form, Social Media + Society 1(2): 1-12.

Orwin, R. G. (1983) A fail-safe N for effect size in meta-analysis, Journal of Educational Statistics 8(2): 157-159.

Paavilainen, J., Hamari, J., Stenros, J. and Kinnunen, J. (2013) Social Network Games: Players’ Perspectives, Simulation \& Gaming 44(6): 794-820.

Paré, G., Trudel, M.-C., Jaana, M. and Kitsiou, S. (2015) Synthesizing information systems knowledge: A typology of literature reviews, Information \& Management 52(2): 183-199.

Park, B.-W. and Lee, K. C. (2011) Exploring the value of purchasing online game items, Computers in Human Behavior 27(6): 2178-2185.

Rosenthal, R. (1979) The "file drawer problem" and tolerance for null results, Psychological Bulletin 86(3): 638-641.

Sabherwal, R., Jeyaraj, A. and Chowa, C. (2006) Information System Success: Individual and Organizational Determinants, Management Science 52(12): 1849-1864. 
Schmidt, F. L. and Hunter, J. E. (1977) Development of a general solution to the problem of validity generalization, Journal of Applied Psychology 62(5): 529-540.

Schmidt, F. L. and Hunter, J. E. (1999) Comparison of three meta-analysis methods revisited: An analysis of Johnson, Mullen, and Salas (1995), Journal of Applied Psychology 84(1): 144-148.

Shin, D. H. (2008) Understanding purchasing behaviors in a virtual economy: Consumer behavior involving virtual currency in Web 2.0 communities, Interacting with Computers 20(4): 433-446.

TechNavio, (2013) Global virtual goods market 2012-2016,

Venkatesh, V., Morris, M. G. Davis, G. B. and Davis, F. D. (2003) User acceptance of information technology: Toward a unified view, MIS Quarterly 27(3): 425-478.

Wang, W.-T. and Chang, W.-H. (2013) The integration of the expectancy disconfirmation and symbolic consumption theories: A case of virtual product consumption, 46th Hawaii International Conference on System Sciences.

Wang, W.-T. and Chang, W.-H. (2014) A study of virtual product consumption from the expectancy disconfirmation and symbolic consumption perspectives, Information Systems Frontiers 16(5): 887-908.

Webster, J. and Martocchio, J. J. (1992) Microcomputer playfulness: Development of a measure with workplace implications, MIS Quarterly 16(2): 201-226.

Webster, J. and Watson, R. T. (2002) Analyzing the Past to Prepare for the Future: Writing a Literature Review, MIS Quarterly 26(2): 13-23. 
Wired Magazine, (2012) These Guys" \$5K Spending Sprees Keep Your Games Free to Play, [Online]

Available at: http://www.wired.com/gamelife/2012/11/meet-the-whales/all/

Yee, N. (2006) The demographics, motivations and derived experiences of users of massivelymultiuser online graphical environments, PRESENCE: Teleoperators and Virtual Environments 15: 309-329. 


\section{APPENDIX}

\section{A. Complete literature search string}

TITLE-ABS-KEY(purchase OR purchasing OR repurchase OR shopping OR buying OR "behavioral outcomes" AND "virtual items" OR "virtual goods" OR "virtual products" OR "digital items" OR "game items" OR "using virtual currency" OR "facebook games" OR "in game purchase" OR "virtual world application" OR "social network games" OR "free-to-play" OR "online games stores" OR "in virtual world")

\section{B. Meta-analysis calculation formulas (Borenstein et al. 2009)}

1. Use $k$ for number of studies and $n$ for sample sizes $n-3$.

2. Fisher z-transform correlations before calculation: $z=\frac{1}{2} \ln \left(\frac{1+r}{1-r}\right)$

3. Estimate between-studies variance: $T^{2}=\frac{Q-d f}{C}$, where

$$
Q=\sum_{i=1}^{k} n_{i} z_{i}^{2}-\frac{\left(\sum_{i=1}^{k} n_{i} z_{i}\right)^{2}}{\sum_{i=1}^{k} n_{i}}, \quad d f=k-1, \quad C=\sum_{i=1}^{k} n_{i}-\frac{\sum_{i=1}^{k} n_{i}^{2}}{\sum_{i=1}^{k} n_{i}}
$$

4. Random effect model weight: $w=\frac{1}{\frac{1}{n}+\tau^{2}}$

5. Magnitude of effect size estimate: $z=\frac{\sum_{i=1}^{k} w_{i} z_{i}}{\sum_{i=1}^{k} w_{i}}$

6. Standard error of effect size estimate: $S E=\sqrt{\frac{1}{\sum w_{i}}}$

7. $95 \%$ confidence intervals of effect size estimate: bounds $=z \pm 1.96 * S E$

8. Statistical significance: $Z=\frac{z}{S E}, \quad p=2(1-\operatorname{NORMDIST}(Z))$

9. Inverse Fisher z-transform back to correlation: $r=\frac{\exp (2 z)-1}{\exp (2 z)+1}$ 


\section{Tests for heterogeneity}

\begin{tabular}{llllll}
\hline Correlation pair & & $\mathrm{Q}$ & $\mathrm{df}(\mathrm{Q})$ & $\mathrm{p}$ & $\mathrm{I}^{2}$ \\
\hline Service Use Enjoyment & x Purchase Intention & 143.455 & 7 & 0.000 & 95.120 \\
Subjective Norms & x Purchase Intention & 204.464 & 7 & 0.000 & 96.576 \\
Flow & x Purchase Intention & 26.509 & 5 & 0.000 & 81.138 \\
Attitude Toward Purchasing & x Purchase Intention & 19.005 & 4 & 0.001 & 78.953 \\
Service Use Intention & x Purchase Intention & 47.628 & 3 & 0.000 & 93.701 \\
Interactivity & x Purchase Intention & 3.859 & 2 & 0.145 & 48.168 \\
Perceived Ease of Use & x Purchase Intention & 23.238 & 2 & 0.000 & 91.393 \\
Perceived Network Size & x Purchase Intention & 21.604 & 2 & 0.000 & 90.743 \\
Perceived Value & x Purchase Intention & 3.981 & 2 & 0.137 & 49.767 \\
Self-Presentation & x Purchase Intention & 15.122 & 2 & 0.001 & 86.774 \\
Social Presence & x Purchase Intention & 18.542 & 2 & 0.000 & 89.214 \\
\hline
\end{tabular}

